\title{
Anosognosia visual en una paciente con lesión isquémica occipital bilateral
}

\section{Visual anosognosia on a patient due to bilateral ischemic occipital lobe strokes}

\author{
Ulises de Dios-Cuadras*, Marco P. Hernández-Abrego, Blanca E. Martínez-Báez \\ y Brenda Y. Juárez-Domínguez \\ Asociación para Evitar la Ceguera en México, Hospital Dr. Luis Sánchez Bulnes, Ciudad de México, México
}

\begin{abstract}
Resumen
Presentación de un caso clínico que trata de una paciente de 60 años de edad que acude a la consulta oftalmológica por disminución de la función visual observada por familiares dos días previos a la consulta; sin embargo, la paciente niega tener alteración de la función visual. A la exploración se encuentra con agudeza visual de movimiento de manos en ambos ojos, sin alteraciones en las estructuras oculares que lo justifiquen. Se recomienda nueva valoración en 24 horas y se solicitan estudios complementarios; sin embargo, la paciente acude una semana después ya con capacidad visual de 20/250 en ambos ojos y con campos visuales manuales, los cuales muestran hemianopsia homónima izquierda y reducción de $30^{\circ}$ periféricos temporales en el ojo derecho y $10^{\circ}$ periféricos nasales en el ojo izquierdo, con conservación parcial del punto de fijación y resonancia magnética de cráneo que muestra lesión isquémica occipitotemporal derecha con isquemia de pequeño vaso occipital bilateral. Se indica tratamiento a cargo de neurooftalmología. Se integra diagnóstico de anosognosia visual asociado a daño isquémico occipital, con mejoría paulatina de la función visual por probable reperfusión del sistema nervioso central.
\end{abstract}

Palabras clave: Isquemia occipital. Anosognosia visual. Síndrome de Anton.

\begin{abstract}
A case report of a 60 year old female brought by her family to medical consultation because they noticed decreased visual function 2 days prior to medical evaluation. The patient denies having altered visual function. Her visual acuity was of hand motion on both eyes with no ocular findings to justify this vision. The patient was told she needed a Goldmann perimetry test and brain magnetic resonance imaging and to be evaluated in 24 hours, nevertheless, the patient returns one week later with a visual capacity of 20/250 in both eyes. By that time, the Goldmann perimetry shows complete left homonymous hemianopsia and peripheral reduction of temporal $30^{\circ}$ on the right eye and nasal $10^{\circ}$ on the left eye with partial preservation of fixation point. Magnetic resonance imaging shows right occipito-temporal ischemic lesion with bilateral small vessel occipital ischemia. Treatment is indicated by neuro-ophthalmology service and the patients were diagnosed with visual anosognosia associated to occipital ischemia, with gradual improvement of visual function by reperfusion of central nervous system.
\end{abstract}

Key words: Occipital ischemia. Visual anosognosia. Anton's Syndrome.

Correspondencia:

*Ulises de Dios-Cuadras

Vicente García Torres, 46

Barrio San Lucas, Del. Coyoacán

Fecha de recepción: 20-04-2017

Disponible en internet: 01-07-2019

C.P. 04030 , Ciudad de México, México

Fecha de aceptación: 07-08-2017

Rev Mex Oftalmol. 2018;93(4):203-208

DOI: 10.24875/RMO.M18000025 www.rmo.com.mx

0187-4519/@ 2017 Sociedad Mexicana de Oftalmología. Publicado por Permanyer México. Este es un artículo Open Access bajo la licencia CC BY-NC-ND (http://creativecommons.org/licenses/by-nc-nd/4.0/). 


\section{Introducción}

Existen causas de baja visual que no se ven relacionadas con patologías oculares y que no son evidentes en una exploración oftalmológica de rutina. En estos casos es importante conocer los posibles sitios de la vía visual que pueden verse comprometidos, así como las diferentes manifestaciones que podemos encontrar en la exploración física para orientar una posible área de afección y, así, conformar una impresión diagnóstica más acertada.

Cuando existe baja visual debido a alteraciones de la vía visual, generalmente va asociada a otro tipo de manifestaciones que en ocasiones no son exteriorizadas por los pacientes hasta que se interrogan de forma dirigida. Pueden incluir cambios específicos en los campos visuales, alteraciones de la percepción de movimientos, variación en la capacidad de observar varios objetos de forma simultánea, incapacidad de reconocer que la visión ha disminuido, incapacidad para reconocer o comprender estímulos visuales, entre otros.

Aproximadamente en el $70 \%$ de casos, las arterias cerebrales posteriores se originan del tronco basilar, en un $20-25 \%$ una lo hace de la carótida y la otra del tronco basilar, y en el resto de casos ambas provienen del sistema carotídeo ${ }^{1}$. Esta variabilidad anatómica puede justificar las múltiples expresiones clínicas que tiene este síndrome vascular. La oclusión ateromatosa de la arteria cerebral posterior es rara, así como también el infarto completo. Se afectan con mayor frecuencia ramas distales, especialmente la calcarina ${ }^{1,2}$. El mecanismo más frecuente es el embólico ${ }^{1}$. La oclusión de ramas temporooccipitales determina alteraciones visuales y trastornos gnósicos ${ }^{2,3}$. Se produce una hemianopsia homónima en un tercio de los casos de oclusión de la arteria cerebral posterior, por afectación del córtex calcarino o estriado y/o de las fibras geniculocalcarinas ${ }^{4}$. En lesiones del hemisferio dominante, la disnomia para colores y la afasia amnésica son más frecuentes que la dislexia sin agrafia ${ }^{5,6}$. La negligencia visual puede ser la manifestación clínica de grandes infartos temporoparietales derechos ${ }^{6}$. No es raro observar perseveraciones visuales asociadas con hemianopsia. La amnesia se observa asociada a lesiones mediales del lóbulo temporal izquierdo, incluyendo a menudo al hipocampo y la sustancia blanca adyacente. La afasia transcortical puede darse en grandes infartos de la región temporooccipital posterior izquierda ${ }^{5,7-9}$.

Un aspecto de interés extraordinario desde el punto de vista clínico lo constituyen los síndromes corticales bilaterales que pueden ocurrir como consecuencia de la oclusión completa de la porción superior del tronco basilar, especialmente si las comunicantes posteriores son incompetentes. Las lesiones occipitales bilaterales ocasionan ceguera cortical o hemianopsia homónima bilateral, asociada a alucinaciones visuales y anosognosia, amnesia y síndrome delirante.

El síndrome de Balint se manifiesta con alteración de la atención espacial, con simultagnosia, ataxia óptica y dificultad para ejecutar movimientos bajo control visual y déficit de generar movimientos oculares hacia objetivos presentes en el campo visual, como una apraxia oculomotora. Se mantienen los movimientos oculares reflejos a estímulos vestibulares, auditivos y a estímulos visuales inesperados. Esta sintomatología es el resultado de infartos cerebrales calcarinos y parietooccipitales bilaterales ${ }^{5,8,9}$. El síndrome de Anton es la situación de ceguera cortical y anosognosia de la misma, en ausencia de demencia o confusión.

La prosopagnosia es un desorden que se caracteriza por la incapacidad de reconocer los rostros. Resulta de lesiones bilaterales, pero la participación del hemisferio occipital derecho parece esencial para su desarrollo $5,8,9$.

Trastorno de la percepción invertida o inclinada. La ilusión de la inversión de la imagen visual consiste en una percepción alterada de la situación de los objetos que se produce por rotaciones completas en los distintos planos del espacio. Es más frecuente en el plano vertical y sobre todo en la isquemia vertebrobasilar. El mecanismo fisiopatológico se produce por una disfunción de la corteza parietal posterior.

No pocos pacientes que sufren un ictus presentan alteraciones visuoespaciales con dificultad para percibir dibujos de objetos superpuestos y para identificar correctamente las agujas del reloj. Estas se deben a lesiones parietooccipitales. La alexia pura es la incapacidad para leer, estando preservados la escritura y el dictado. Está alterada también la copia. La topografía lesional suele ser la paraventricular al asta occipital del ventrículo lateral izquierdo. La agnosia visual se produce por lesiones bilaterales del sector subcalcarino. Las lesiones del lóbulo occipital inferior u occipitotemporales afectan a estructuras de la región ventral del córtex visual asociativo, $0^{5,8,9}$.

\section{Caso clínico}

Se trata de una paciente de 60 años de edad originaria y residente de la ciudad de México, dedicada a las labores del hogar, que acude a consulta de urgencia de oftalmología traída por su hijo, quien observa 
que la paciente choca con objetos al caminar, con evolución de dos días previos a la valoración. La paciente niega síntomas visuales, al interrogarla directamente refiere tener buena visión e incluso procura, sin éxito, describir la vestimenta del médico como muestra de su capacidad visual y se la observa convencida de haberlo hecho correctamente.

Como antecedentes familiares refiere tener padres con diagnóstico de diabetes mellitus y dentro de los antecedentes personales patológicos presenta hipertensión arterial de 20 años de diagnóstico en tratamiento con losartán, se desconoce la dosis. Resto de antecedentes negados.

A la exploración neurológica, las funciones mentales se encuentran conservadas, con correcta ubicación en persona, tiempo y espacio. Nervios craneales sin alteraciones, sistema motor y sensitivo normal.

A la exploración oftalmológica se encuentran los siguientes hallazgos: Ojo derecho con visión de movimiento de manos, presión intraocular de $12 \mathrm{mmHg}$, párpados y anexos normales, conjuntiva clara, córnea transparente, pupila reactiva, con reflejos normales, cristalino con opacidad NO2NC2, retina aplicada, con cruces arteriovenosos, disminución del calibre arteriolar, mácula con brillo y papila con excavación $5 / 10$ (Fig. 1). Ojo izquierdo con visión de movimiento de manos, presión intraocular de $13 \mathrm{mmHg}$, párpados y anexos normales, conjuntiva clara, córnea transparente, pupila reactiva, con reflejos normales, cristalino con opacidad NO2NC2, retina aplicada, con cruces arteriovenosos, disminución del calibre arteriolar, mácula con brillo y papila con excavación 5/10 (Fig. 2).

Se realiza refracción de la paciente por baja visual en estudio y se encuentran los siguientes datos: en el ojo derecho se observa $-2.25-0.50 \times 15^{\circ}$ con capacidad visual de cuenta dedos a $10 \mathrm{~cm}$, y en el ojo izquierdo $-2.00-1.75 \times 15^{\circ}$ con capacidad visual de movimiento de manos.

Se indica valoración en 24 horas. Se solicitan campos visuales manuales y resonancia magnética nuclear de cráneo para complementar la valoración. La paciente tarda una semana en realizar los estudios solicitados y acudir a la valoración. Se presenta con capacidad visual de 20/250 en ambos ojos sin cambios en la exploración física.

En los campos visuales manuales se observa hemianopsia homónima izquierda completa y reducción de $30^{\circ}$ periféricos temporales en el ojo derecho y $10^{\circ}$ periféricos nasales en el ojo izquierdo, con conservación parcial de punto de fijación (Fig. 3).

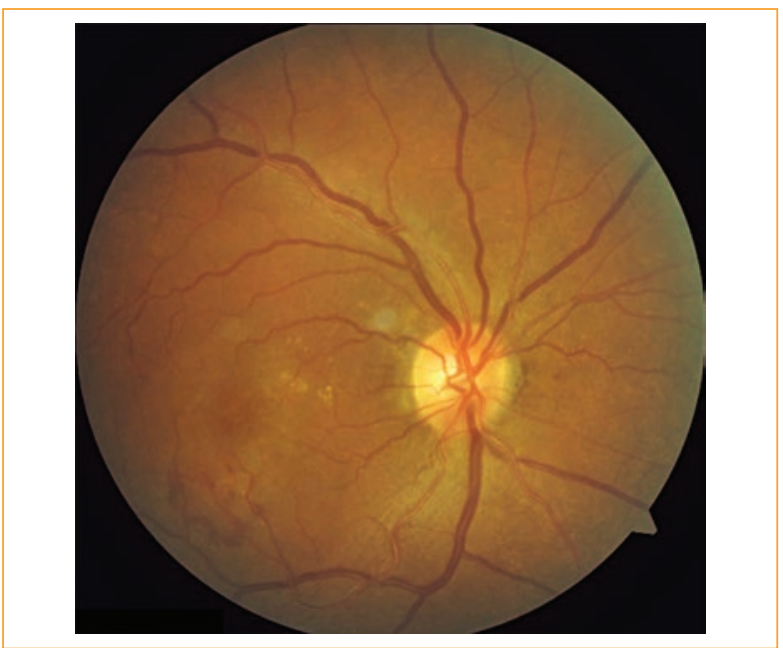

Figura 1. Imagen de segmento posterior ojo derecho.

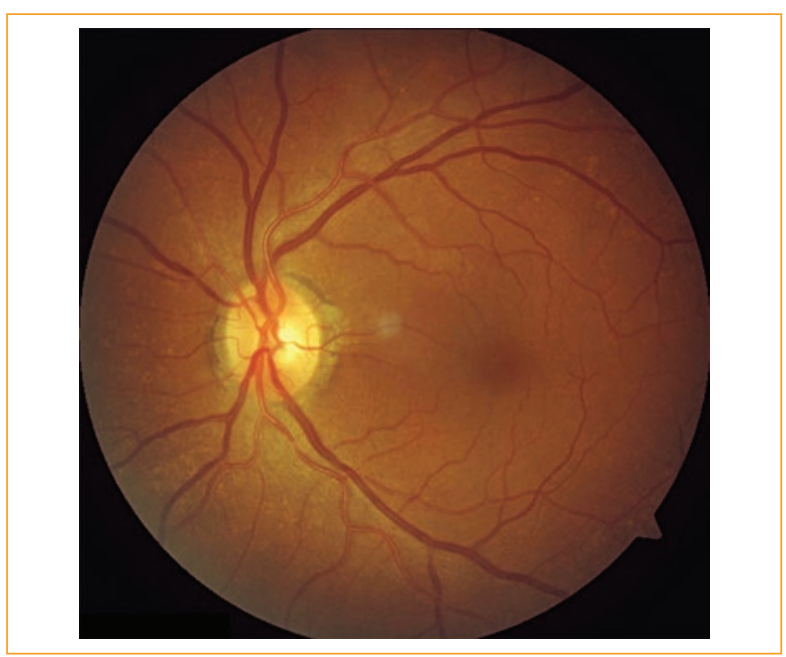

Figura 2. Imagen de segmento posterior ojo izquierdo.

En la resonancia magnética nuclear es posible observar cortes axiales con lesión isquémica occipitotemporal derecha, con isquemia de pequeño vaso occipital bilateral (Fig. 4) (Fig. 5) y cortes coronales donde se observa un defecto cortical del polo occipital derecho (Fig. 6).

Se decide indicar tratamiento a cargo del servicio de neurooftalmología del hospital con atenolol $50 \mathrm{mg}$ cada $24 \mathrm{~h}$, espironolactona $50 \mathrm{mg}$ cada $24 \mathrm{~h}$, amlodipino $5 \mathrm{mg}$ cada $24 \mathrm{~h}$, atorvastatina $20 \mathrm{mg}$ cada $24 \mathrm{~h}$ y se refiere a valoración por neurología.

En la evolución de la paciente a dos meses del ingreso se encuentra con una capacidad visual de 20/100 en ambos ojos sin cambios a la exploración oftalmológica ni en la 


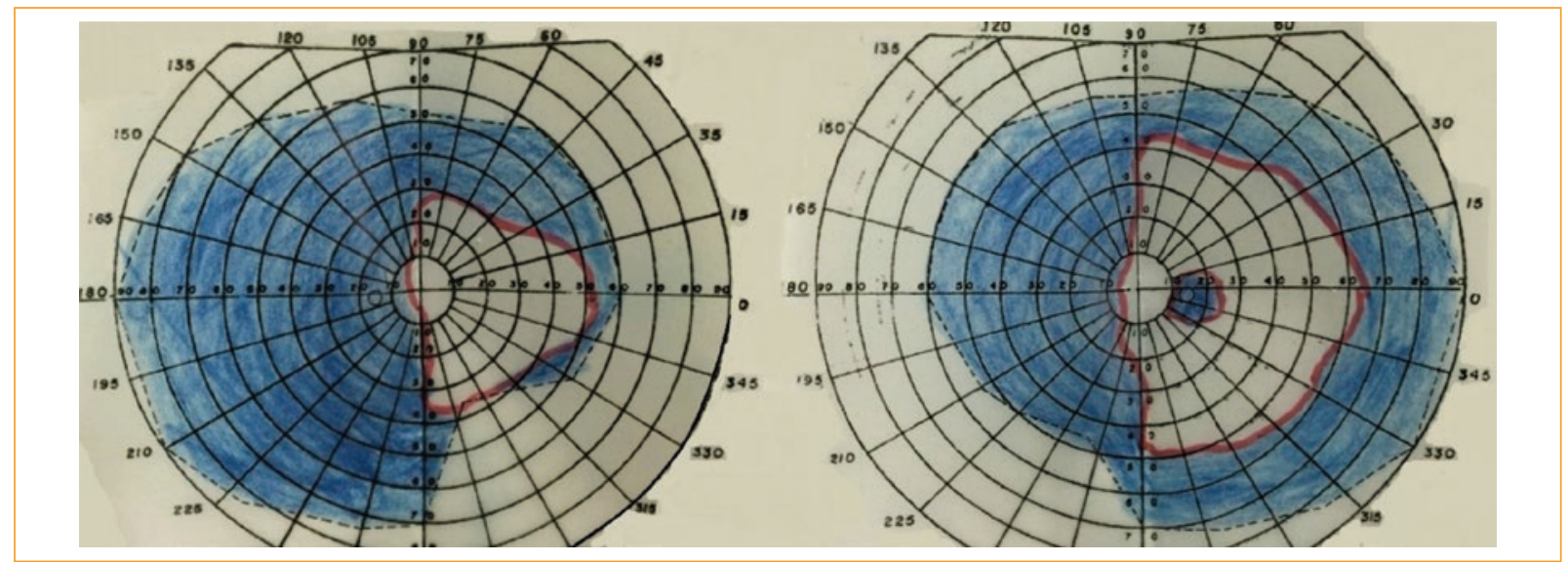

Figura 3. Perimetría.

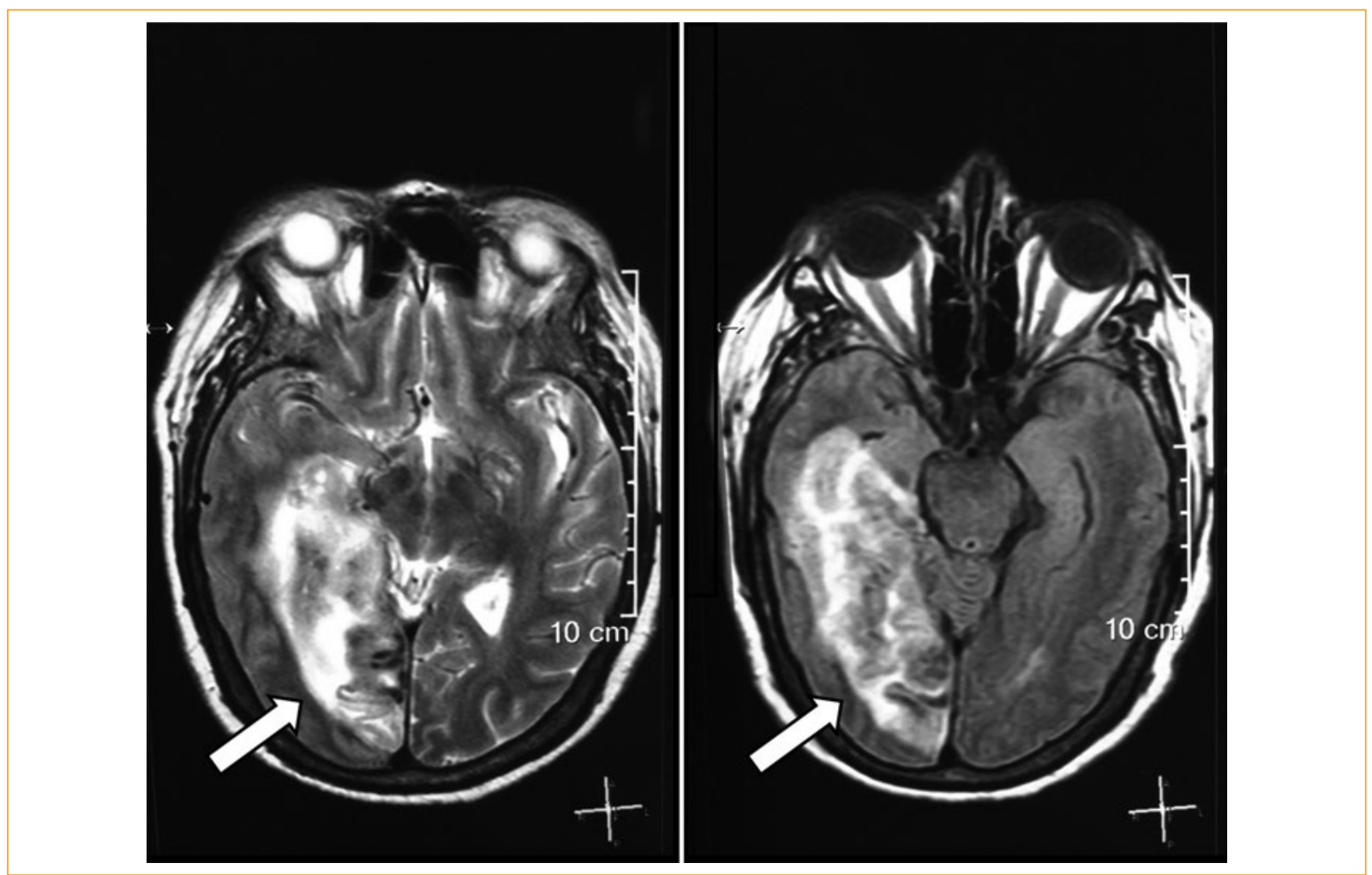

Figura 4. Resonancia magnética nuclear cortes axiales.

sintomatología. Los familiares refieren que aún choca con los objetos.

\section{Discusión}

En el momento del primer contacto con la paciente llama la atención que no refiere problemas en la función visual. Ella acude traída por sus familiares porque ellos son quienes observan que la paciente ha perdido la capacidad de ver y reconocer objetos. Es en ese momento en el que se llega a la deducción de que la paciente tiene una alteración neurológica, la cual se clasifica como anosognosia visual o síndrome de Anton. 
La mala capacidad visual de la paciente no puede ser justificada por ninguno de los cambios oculares que presenta. Es por eso que se decide realizar estudios complementarios, como campos visuales y estudios de imagen del sistema nervioso central. Los campos visuales muestran cambios que se correlacionan con los datos de lesión isquémica en la corteza occipital en la resonancia magnética de nuestra paciente.

La mala capacidad visual que encontramos al ingreso de la paciente contrasta con una aparente conservación del punto de fijación una semana después al realizar los campos visuales. Esto puede explicarse por reperfusión del área de integración macular por circulación colateral. Esta posibilidad de reperfusión se basa en una evolución

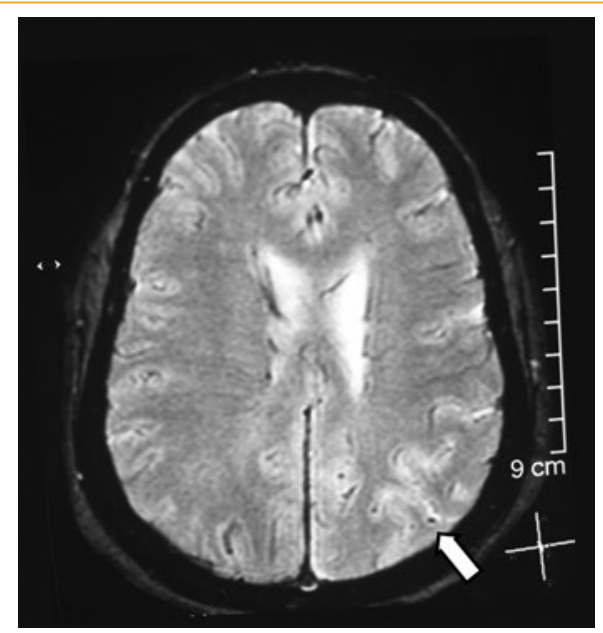

Figura 5. Resonancia magnética nuclear con datos de isquemia por enfermedad de pequeño vaso en región parietotemporooccipital izquierda (flecha). clínica hacia la mejoría; sin embargo, para poder confirmar dicho fenómeno de reperfusión, idealmente se debe realizar un segundo estudio de resonancia magnética con secuencia de difusión. En nuestra paciente no fue posible realizarlo por motivos económicos.

\section{Conclusión}

La vía visual puede verse afectada en cualquiera de sus regiones por lesiones de tipo isquémico dando manifestaciones muy variadas que deben tomarse en cuenta al enfrentarse con un caso de baja visual de origen cortical. Es importante conocer que no todos los casos de baja visual tienen origen en el globo ocular, ya que, reconocer a tiempo patologías con otras manifestaciones visuales asociadas puede tener un impacto positivo en el diagnóstico oportuno de lesiones del sistema nervioso central. Es importante también tomar en cuenta las comorbilidades que pueden estar presentes en pacientes con este tipo de alteraciones, ya que gran parte del manejo se ve enfocado a un control metabólico estricto y a un estrecho seguimiento con un equipo interdisciplinario para una valoración y tratamiento integral.

\section{Responsabilidades éticas}

Protección de personas y animales. Los autores declaran que para esta investigación no se han realizado experimentos en seres humanos ni en animales.

Confidencialidad de los datos. Los autores declaran que han seguido los protocolos de su centro de trabajo sobre la publicación de datos de pacientes.

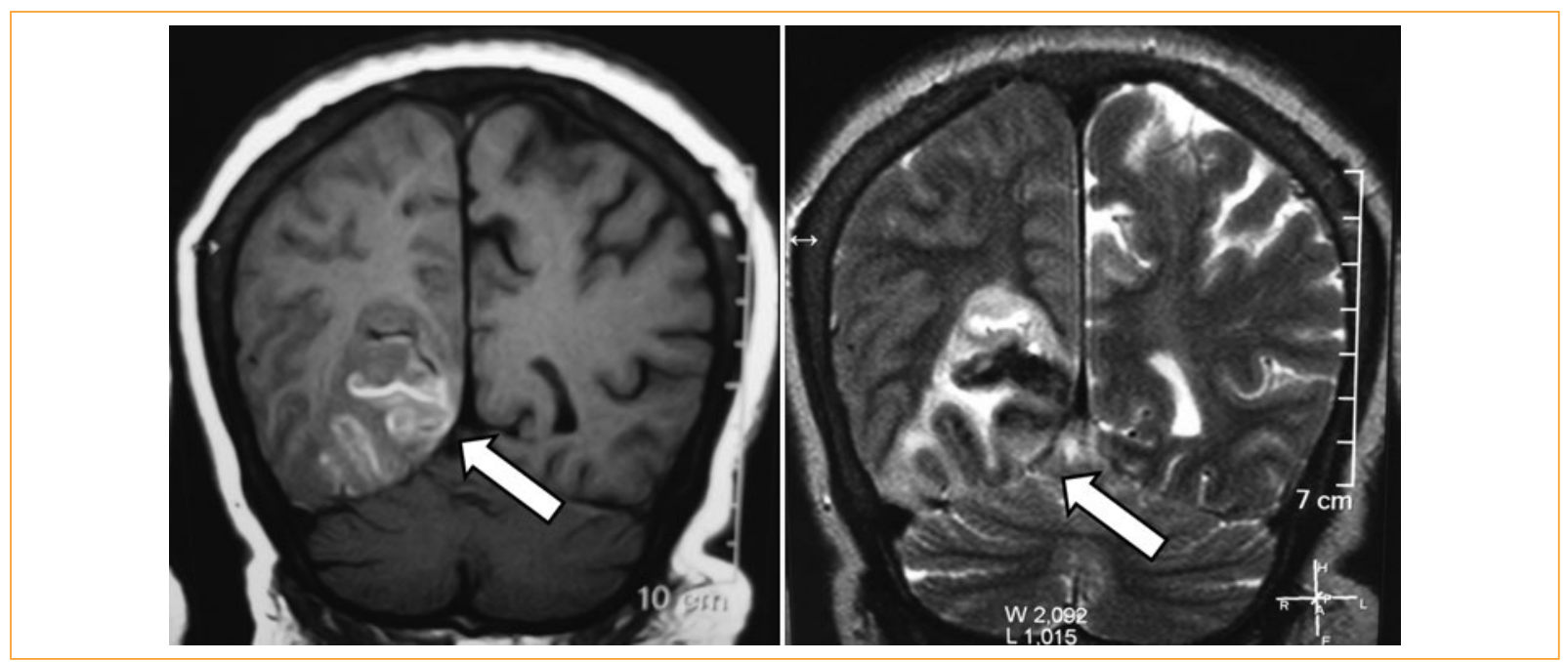

Figura 6. Resonancia magnética nuclear cortes coronales. 
Derecho a la privacidad y consentimiento informado. Los autores declaran que se obtuvo autorización escrita por parte de la paciente para publicación de sus estudios de gabinete y datos clínicos. En este articulo no se revela ningún dato de carácter personal.

\section{Financiamiento}

Los autores declaran que no se obtuvo financiación para esta publicación.

\section{Conflicto de intereses}

Los autores declaran que no existe conflicto de intereses.

\section{Bibliografía}

1. WirtschaLter JD. Anatomic basis and differential diagnosis of field defects. En: Walsh TJ. Visual Fields. Examination and interpretation. Ophthalmology monograps 3. San Francisco: American Academy of Ophthalmology; 1996. p. 39-83.

2. Wall M, Punke SG, Stickney TL, Brito CF, Withrow KR, Kardon RH. SITA standard in optic neuropathies and hemianopias: a comparison with full threshold testing. Invest Ophthalmol Vis Sci. 2001;42:S28-37.

3. Kerrison JB, Lynn MJ, Baer CA, Newman SA, Biousse V, Newman NJ. Stages of improvement in visual fields after pituitary tumor resection. Am J Ophthalmol. 2000;130:813-20.

4. Wong AMF, Sharpe JA. A comparison of tangent screen, Goldmann, and Humphrey perimetry in the detection and localization of occipital lesions. Ophthalmology. 2000;107:527-44.

5. Bell RA, Thompson HS. Relative afferent pupillary defect in optic tract hemianopsias. Am J Ophthalmo. 1978;85:538-40.

6. Benton S, Levy I, Swash M. Vision in the temporal crescent in occipital infarction. Brain. 1980;103:83-97.

7. Borruat FX, Maeder P. Sectoranopia after head trauma: Evidence of lateral geniculate body lesion on MRI. Neurology. 1995;45:590-2.

8. García R, Moreno Ramos T. Neuropatías ópticas: revisión sindrómica y etiológica. Neurol. 2007:3 Supl. 8:16-26.

9. Disorders of Vision. Continuum: Lifelong Learning in Neurology. Neuro-ophthalmology Update. 2003;9:11-78. 